\title{
Articles
}

The Person and the Challenges

Volume 1 (2011) Number 2, p. 11-19

Józef Stala

The Pontifical University of John Paul II in Cracow, Poland

\section{Aspekte der Aktivitäten der akademischen Mitarbeiter an der Theologischen Fakultät, Sektion Tarnów, im Dienst der Wissenschaft}

\author{
Aspects of Activities in the Service of Science \\ by Academic Staff of the Theology Faculty, Section in Tarnów
}

\section{Abstract}

The aim of the Faculty of Theology, Section in Tarnów of the Pontifical University of John Paul II in Cracow is a comprehensive and systematic study of God's revelation in different periods and aspects of the life of the Church and a human being. In this article the author presents various aspects of scientific and didactic work undertaken in the service of science by the academic staff of the Faculty of Theology, Section in Tarnów. First, an acoount will be given of the scientific contacts (meetings, consultations, conferences, seminars, reviews) with the academics of various scientific and research institutions, both from Poland and abroad. Then, the new book series (nearly two hundred books in total), scientific journals and various publications will be highlighted.

\section{Keywords}

Faculty of Theology Section in Tarnów, academic staff, scientific cooperation, publications. 
Nach dem Statut der Päpstlichen Universität Johannes Paul II. in Krakau setzt die „Theologische Fakultät, Sektion Tarnów, die Arbeit des Theologischen Instituts in Tarnów fort. Das Spezifikum der Fakultät findet in ihrer kirchlichen Rechtsform und in ihrer Organisationsstruktur seinen Ausdruck, insbesondere in der Person und den Kompetenzen des Großen Vizekanzlers sowie in ihrer Bedeutung für Leben und Mission der Kirche von Tarnów. Das Ziel der Fakultät besteht in einer allseitigen und systematischen Erforschung der Göttlichen Offenbarung in den unterschiedlichen Epochen und Ausprägungen des Lebens der Kirche und des Menschen". Darum erscheint es nur zu berechtigt, in dem vorliegenden Artikel Aspekte der Aktivitäten an der Theologischen Fakultät, Sektion Tarnów, aufzugreifen und zu präsentieren, welche die akademischen Mitarbeiter im Dienst der Wissenschaft aufnehmen. Zunächst werden die wissenschaftlichen Kontakte (Zusammenkünfte, Konsultationen, Rezensionen, Seminare, Konferenzen) dargelegt, die bereits mit akademischen Mitarbeitern von Hochschulen sowohl in Polen als auch im Ausland geknüpft wurden. Daran schließt sich die Charakterisierung von annähernd zwanzig Verlagsserien an, zu denen fast 200 Buchveröffentlichungen zählen, bevor die wissenschaftlichen Periodika besprochen werden, die ebenfalls an der Fakultät herausgegeben werden.

\section{Die Zusammenarbeit mit Hochschulen im In- und Ausland}

Die Theologische Fakultät, Sektion Tarnów, hat zwar bisher noch keine Forschungsaufgaben im Auftrag einer anderen Hochschule durchgeführt, die akademischen Mitarbeiter an der Fakultät arbeiten jedoch im Rahmen der Tätigkeit der einzelnen Lehrstühle bereits mit Hochschulen im In- und Ausland zusammen. Viele Mitarbeiter der Fakultät unterhalten im Zusammenhang mit den Arbeiten der einzelnen Lehrstühle wissenschaftliche Forschungskontakte in Form von Erfahrungs- und Literaturaustausch, Teilnahmen an wissenschaftlichen Konferenzen, Konsultationen in Bezug auf wissenschaftliche Forschungen, die von den Mitarbeitern durchgeführt werden, gemeinsamer Projektierung von Forschungsvorhaben sowie Rezensionen akademischer Arbeiten an zahlreichen Hochschulen in Polen. Darüber hinaus werden weitere Forschungskontakte geknüpft, die überwiegend den Austausch von Quellenliteratur und wissenschaftlichen Informationen sowie die Teilnahme an wissenschaftlichen Konferenzen, Vorträgen und Konsultationen mit Hochschulen im Ausland

\footnotetext{
${ }^{1}$ Statut der Päpstlichen Universität Johannes Paul II. in Krakau, Nr. 143-144.
} 
betreffen (unter anderem: Universität in Ljubljana - Slowenien, Universität in Pamplona - Spanien, Universität Santa Croce in Rom - Italien, Karlsuniversität in Prag - Tschechien, Katholische Universität in Lissabon - Portugal, Universite Katholikue de Louvain - Belgien, Katholieke Universiteit Leuven - Belgien, Katholische Universität in Ruzomberok - Slowakei, Instituto di Ricerca sul Volto di Cristo in Rom, Priesterseminar in Gródek Podolski - Ukraine). Im Rahmen des „Erasmus” - Programms hat die Theologische Fakultät, Sektion Tarnów, bilaterale Vereinbarungen mit folgenden Hochschulen unterzeichnet: Theologische Fakultät derUniversitätinLjubljana-Slowenien, TheologischeFakultätderKarlsuniversität in Prag - Tschechien, Pädagogische Fakultät der Katholischen Universität in Ruzomberok - Slowakei und Theologische Fakultät der Katholischen Universität in Lissabon - Portugal. Am Austausch nehmen akademische Mitarbeiter und Studenten teil. Akademische Mitarbeiter der Fakultät haben sich ebenso bereits an den Arbeiten vieler Vereinigungen und Organisationen auf dem Gebiet der Theologie und des Erziehungs- und Bildungswesens beteiligt. $\mathrm{Zu}$ einer derartigen Zusammenarbeit verpflichtet auch das Statut der Päpstlichen Universität Johannes Paul II. in Krakau, wo es heißt: „Die Päpstliche Universität Johannes Paul II. in Krakau nimmt gemäß der jahrhundertealten Tradition der Theologischen Fakultät der Jagiellonen-UniversitätdieZusammenarbeitimBereich vonwissenschaftlichen Forschungen, didaktischer Arbeit und Verlagsveröffentlichungen mit anderen Hochschulen und wissenschaftlichen Instituten im In- und Ausland auf... Auf der Grundlage gegenseitiger Vereinbarungen bezieht sich diese Zusammenarbeit auf: die Durchführung gemeinsamer wissenschaftlicher Forschungen, den Austausch akademischer Mitarbeiter, Verwaltungsangestellter und Studenten, die Zusammenarbeit bei Verlagsangelegenheiten und den Austausch von Büchern und Wissenschaftszeitschriften, die Teilnahme und die Organisation von gemeinsamen Symposien und Wissenschaftskongressen"2.

\section{Verlagsserien}

Die akademischen Mitarbeiter der Theologischen Fakultät, Sektion Tarnów, der Päpstlichen Universität Johannes Paul II. in Krakau veröffentlichen die Ergebnisse ihrer wissenschaftlichen Forschungsarbeiten in folgenden systematisch erscheinenden Serien”: „Academica”, „Rozprawy naukowe” (Wissenschaftliche Debatten), „Edukacja dzisiaj. Problemy i wyzwania” (Erziehen heute. Probleme

\footnotetext{
${ }^{2}$ Statut der Päpstlichen Universität Johannes Paul II. in Krakau, Nr. 131-132.

${ }^{3}$ Die Auflistung entspricht dem Stand vom 30. Juni 2011.
} 
und Herausforderungen), „Quaestiones ad disputandum”, „Ogólnopolskie Forum Sądowe” (Landesweites Gerichtsforum), „Sądecka Góra Tabor” (Berg Tabor in Nowy Sacz), „Thologumena”, „Bibliotheca Tarnoviensis”, „W kręgu Słowa” (Im Kreis des Wortes), „Krąg Biblijny” (Bibelkreis), „Poznając Biblię” (Die Bibel kennenlernen), „Duszpasterski Komentarz do Nowego Testamentu” (Seelsorgerlicher Kommentar zum Neuen Testament), „Scripturae Lumen” und „Formacja socjalna” (Soziale Bildung). Die nahezu 200 Buchveröffentlichungen, die seit dem Beginn der angeführten Reihen erschienen sind, haben in der Mehrzahl eine wissenschaftliche Ausprägung, wobei einige auch überwiegend die Seelsorge betreffen.

Die Reihe „Academica” ist eine Sammlung lehrbuchartiger Synthesen aus den Gebieten Theologie, Philosophie, Biblistik und Psychologie, die sich an die Studenten theologischer und humanistischer Hochschulen wie auch an Leser mit vertieftem Interesse für diesen Fachbereich richtet. Bisher sind in der Reihe „Academica” 83 Bände erschienen. In der Serie „Rozprawy naukowe” werden aktuell durchgeführte wissenschaftliche Forschungen aus den Bereichen Philosophie und Theologie vorgestellt, aus denen Habilitationsschriften resultieren. Die Adressaten dieser Serie sind vor allem akademische Mitarbeiter, bis heute sind elf Bücher herausgegeben. In der Reihe „Edukacja dzisiaj. Problemy i wyzwania” werden die bedeutendsten Herausforderungen an Katechese, Erziehung, Bildung und Pädagogik aufgegriffen, die mit den gewaltigen gesellschaftlichen, kulturellen und religiösen Umbrüchen verbunden sind, die sich in Europa vollziehen. Die Reihe präsentiert sowohl theoretische Grundsätze als auch praktische Lösungen, die mit der Realisierung des didaktischen und katechetischen Prozesses sowie mit Erziehung und Bildung in Verbindung stehen. Die publizierten Problemkreise können insbesondere Studenten und Dozenten der Theologie, der Pädagogik, der Erziehungswissenschaften und der humanistischen Wissenschaften hilfreich sein, den Theoretikern dabei ebenso wie den Praktikern. Die Reihe „Edukacja dzisiaj. Problemy i wyzwania” umfasst bisher 23 Bände. In der Serie „Quaestiones ad disputandum"werden vor allem die Resultate wissenschaftlicher Forschungen dargelegt, die in Symposien und Wissenschaftskonferenzen aufgenommen wurden. Bis heute sind in der Serie „Quaestiones ad disputandum” elf Bücher erschienen. Die Reihe „Ogólnopolskie Forum Sądowe” enthält Materialien aus den Zusammenkünften der Mitarbeiter der kirchlichen Gerichtsbarkeit, die etwa im Abstand von zwei Jahren in Gródek nad Dunajcem stattfinden. Die Referate der Experten für Kanonisches Recht aus Polen und der Mitarbeiter der Apostolischen Gerichte befassen sich mit Problemen, die mit dem Ehenichtigkeitsverfahren in Verbindung stehen, insbesondere werden Themen zum materiellen Eherecht und 
zum kirchlichen Verfahrensrecht aufgegriffen. In der Reihe „Ogólnopolskie Forum Sądowe" sind bis jetzt vier Bände aufgelegt. Die Serie "Sądecka Góra Tabor” ist der Geschichte von Frömmigkeit und Geistlichkeit in der Gegend um Nowy Sącz gewidmet. Sie publiziert Quellenmaterial zum religiös-geistlichen Leben in dieser Region, die bisher noch nicht Gegenstand separater Untersuchungen waren, ebenso wie Berichte über Sanktuarien und Wallfahrtszentren, die für die Ausprägung der Frömmigkeit dort eine vorrangige Rolle spielen. Zum jetzigen Zeitpunkt umfasst die Serie „Sądecka Góra Tabor" fünf Bücher. Die Reihe „Thologumena” greift aktuelle theologische Probleme auf und entsteht überwiegend auf der Grundlage organisierter Symposien, insbesondere in der Zusammenarbeit mit den Mitgliedern der "Polskie Towarzystwo Teologiczne Oddział w Tarnowie" (Theologische Gesellschaft Polen, Abteilung Tarnów). In der Reihe "Thologumena" sind bisher sechs Buchveröffentlichungen erschienen. Die Serie „Bibliotheca Tarnoviensis” widmet sich vor allem dem Erbe der Kirchengeschichte, wobei der Schwerpunkt auf der Diözese Tarnów liegt. Dabei geht es um den Ausbau der bislang recht schwach entwickelten Historiographie der Kirche in Tarnów. Sie umfasst ebenso Arbeiten, die sich mit der Geschichte der Kirche in Tarnów befassen, wie Forschungen in Verbindung mit der Diözese Tarnów, insbesondere aus der Theologischen Fakultät, Sektion Tarnów, der UPJPII. Bisher gibt es aus der Serie „Bibliotheca Tarnoviensis” neun Buchveröffentlichungen. Die Reihe „W kręgu Słowa” erörtert Problemstellungen aus den Bereichen der Bibelexegese und der Bibeltheologie. Sie setzt sich aus monographischen Arbeiten aus dem Gebiet des Alten und des Neuen Testaments zusammen, die ausgewählte Themen und Fragen analysieren. Die Reihe „W kręgu Słowa" besteht seit 2006 und umfasst heute zehn Bände. Des weiteren erscheinen die Bücher der Serie „Krag Biblijny” dreimal im Jahr. Sie gliedern sich in zwei Teile, wobei der erste zur biblischen Prophoristik zu zählen ist. Im Geist der Methode von „Krąg Biblijny” werden einzelne biblische Perikope analysiert. Der zweite Teil besteht aus einer Abhandlung aus dem Bereich der allgemeinen Einführung in die Heilige Schrift, der Einführung in das Alte und auch das Neue Testament. Diese Bücher erscheinen in Verbindung mit dem „Dzieło Biblijne im. Jana Pawla II" (Bibelwerk im Namen Johannes Pauls II.). Aus dieser Serie gibt es bisher 15 Ausgaben. Dagegen sind die Bücher aus der Reihe „Poznając Biblię” eine schriftliche Aufzeichnung der Vorlesungen, die im Rahmen des „RadiowoInternetowe Studium Biblijne" (Bibelstudium im Radio oder im Internet) gehalten werden. Einzelne Teile kann man als Lehrwerke bezeichnen, die Grundlagen der allgemeinen und speziellen Einführung in das Alte und das Neue Testament enthalten. Bislang sind in der Reihe „Poznając Biblię” vier Bücher erschienen. 
Die Serie „Duszpasterski Komentarz do Nowego Testamentu” ist Ausdruck der Notwendigkeit einer prophoristischen Betrachtung der Bibel und publiziert monographische Ausarbeitungen zu einzelnen Schriften des Neuen Testaments. Die Serie „Duszpasterski Komentarz do Nowego Testamentu” umfasst derzeit vier Bücher. Seit drei Jahren erscheint die Reihe „Scripturae Lumen”. Nach Absicht der Autoren präsentieren einzelne Bände breit gefasste monographische Arbeiten zu ausgewählten theologischen Themen. Zur Mitarbeit sind Theologen und Exegesen aus unterschiedlichen Forschungsbereichen eingeladen. Bis heute sind in der Reihe „Scripturae Lumen” drei Bücher erschienen. Der Glaube schließt sich nicht im Menschen ein sondern strahlt auf die Umgebung ab und verändert sie wie der biblische Sauerteig den Kuchen. In diesem Licht ergeben sich unablässig aktuelle Fragen über die Verpflichtung der Jünger Jesu - der Christen - im Kontext ihres Engagements und ihres Eintretens für das Leben der Welt. Daher werden unter anderem Fragen nach dem Allgemeinwohl, nach Hilfsleistungen mit der Grundeinstellung zur Hilfsbereitschaft, nach Solidarität und Liebe wichtig sein, was für die soziale Arbeit und Bildung von außerordentlich wesentlicher Bedeutung ist. Deshalb werden in der Reihe „Formacja socjalna” Probleme aufgegriffen, die mit der Bildung in ihrer sozialen Dimension verbunden sind, denn die Treue zur Lehre Christi steht in enger Verbindung mit dem Leben der Gesellschaft, mit dem Engagement auf sozialer Ebene. Die Reihe „Formacja socjalna" umfasst bislang drei Bücher.

\section{Wissenschaftliche Periodika}

Die akademischen Mitarbeiter der Theologischen Fakultät, Sektion Tarnów, geben folgende wissenschaftliche Periodika heraus: „Tarnowskie Studia Teologiczne" (Theologische Studien in Tarnów), "The Person and the Challenges: The Journal of Theology, Education, Canon Law and Social Studies Inspired by Pope John Paul II" und ebenso „Studia Regionalne” (Regionale Studien). Darüber hinaus ist in den Jahren von 1947 bis 2001 das Periodikum „Religioni et Litteris” erschienen.

Die „Tarnowskie Studia Teologiczne” sind eine rezensierte wissenschaftliche Zeitschrift, die seit 1938 halbjährlich in polnischer Sprache von den akademischen Mitarbeitern der Theologischen Fakultät, Sektion Tarnów, der Päpstlichen Universität Johannes Paul II. in Krakau herausgegeben werden. Die ersten vier Ausgaben aus den Jahren 1938 und 1939 publizierten wissenschaftliche Erörterungen. Der Ausbruch des Zweiten Weltkriegs führte zur Einstellung des 
Periodikums. Im Jahr 1972 wurde die Zeitschrift erneut aufgelegt und enthielt dann Texte von akademischen Mitarbeitern des Instituts in Tarnów, aber auch von außerhalb. In den Jahren von 1972 bis 1983 wurden fünf Ausgaben veröffentlicht. Seit 1986 erschien das Periodikum regelmäßig, zunächst einmal im Jahr und seit 1998 halbjährlich. Neben Artikeln umfasst es übersetzte Texte und Kommentare dazu, Rezensionen von Publikationen aus dem In- und Ausland sowie Berichte über Symposien. Unter den mehr als 40 Bänden, die bisher publiziert wurden, finden sich auch einige Sonderausgaben und Schriften, die Priestern aus der Diözese Tarnów gewidmet sind.

Das internationale Periodikum "The Person and the Challenges: The Journal of Theology, Education, Canon Law and Social Studies Inspired by Pope John Paul II", eine rezensierte wissenschaftliche Zeitschrift, ist mit dem Ziel entstanden, internationale Forschungen aus den Bereichen Theologie, Bildung, Kanonisches Recht und Sozialwissenschaften zu verbreiten, die von der Person und der Lehre Papst Johannes Pauls II. inspiriert sind, indem die Diskussion um Fragen aus den oben angeführten und weiteren verwandten Gebieten angestoßen wird. In "The Person and the Challenges" sind wissenschaftlich beachtliche, bisher unveröffentlichteArbeiten abgedruckt: Artikel, wie auch Berichte von bedeutenden Konferenzen und Buchrezensionen. Die Texte (in englischer, deutscher, spanischer, italienischer und französischer Sprache) sind als Papierversion und in digitaler Form (als PDF) auf der Internetseite der Zeitschrift publiziert. Jeder Artikel, der in einer der fünf angeführten Sprache geschrieben ist, enthält auch folgendes in englischer Sprache: Überschrift, Schlüsselwörter und eine kurze Zusammenfassung. Das Periodikum wird ab 2011, dem Jahr der Seligsprechung des Heiligen Vaters Johannes Paul II. an der Theologischen Fakultät, Sektion Tarnów, der Päpstlichen Universität Johannes Paul II. in Krakau herausgegeben. Der Redaktion und dem wissenschaftlichen Beirat gehören Wissenschaftler aus der ganzen Welt an.

Die „Studia Regionalne” sind ein wissenschaftliches Jahrbuch, das seit 2007 erscheint. Die „Studia Regionalne” sind als regional zu charakterisieren und führen Untersuchungen theoretischer Art durch, das heißt sie umfassen Reflexionen über die Region als Kategorie des Lebens und Denkens. Als regional sind sie im historisch-kulturellen Sinn zu bezeichnen, was bedeutet, dass Artikel bevorzugt werden, die der Region Mielec (Geschichte, Menschen, Kultur, Ökonomie) gewidmet sind oder auch einer Region und einem Sachverhalt, die in irgendeiner Weise - sogar weitläufig - mit Mielec und seiner Umgebung in Verbindung stehen. Die „Studia Regionalne” erscheinen in der Zusammenarbeit mit der wissenschaftlichen „Polskie Towarzystwo Teologiczne Oddział w Tarnowie” 
(Theologische Gesellschaft Polen, Abteilung Tarnów). Diese Zusammenarbeit besteht vor allem in meritorischen Konsultationen (Rezensionen) und bietet eine Plattform, auf der die Forschungsergebnisse aus dem für die „Studia Regionalne” spezifischen Bereich präsentiert werden können.

Die verschiedenen Aktivitäten, die von den akademischen Mitarbeitern der Theologischen Fakultät, Sektion Tarnów, aufgenommen werden und sich unter anderem in wissenschaftlichen Kontakten (Zusammenkünfte, Konsultationen, Rezensionen, Seminare, Konferenzen) mit akademischen Mitarbeitern von Hochschulen sowohl in Polen als auch im Ausland ebenso ausdrücken wie in nahezu 200 Buchveröffentlichungen im Rahmen von annähernd zwanzig Bücherreihen und den herausgegebenen wissenschaftlichen Periodika, zeugen von dem Bemühen um die Weiterentwicklung der Wissenschaft. Man kann dies als ihre Art von Versuch bezeichnen, die Gesellschaft, die Kultur und die Bereiche der Politik und der Wirtschaft für Christus zu öffnen, so wie es er Heilige Vater Johannes Paul II. getan hat, und woran Benedikt XVI. am 1. Mai 2011 in seiner Predigt anlässlich der Seligsprechung Johannes Pauls II. auf dem Petersplatz im Vatikan erinnert hat: „In seinem Testament schrieb der neue Selige: `Als das Konklave der Kardinäle am 16. Oktober 1978 Johannes Paul II. wählte, sagte der polnische Primas Kardinal Stefan Wyszyński zu mir: 'Die Aufgabe des neuen Papstes wird es sein, die Kirche ins Dritte Jahrtausend zu führen` Und weiter führte er aus: 'Ich möchte noch einmal Dankbarkeit gegenüber dem Heiligen Geist für das große Geschenk des Zweiten Vatikanischen Konzils zum Ausdruck bringen, als dessen Schuldner ich mich gemeinsam mit der ganzen Kirche - und vor allem mit dem gesamten Episkopat - fühle. Ich bin überzeugt, dass es den neuen Generationen noch lange aufgegeben sein wird, die Reichtümer auszuschöpfen, die dieses Konzil des 20. Jahrhunderts uns geschenkt hat. Als Bischof, der an dem Konzilsgeschehen vom ersten bis zum letzten Tag teilgenommen hat, möchte ich dieses große Erbe all jenen anvertrauen, die in Zukunft gerufen sein werden, es zu verwirklichen. Ich selbst aber danke dem Ewigen Hirten dafür, dass er mir erlaubt hat, dieser großartigen Sache während all der Jahre meines Pontifikats zu dienen.' Und was ist diese „Sache“? Es ist dieselbe, die Johannes Paul II. in seiner ersten feierlichen Messe auf dem Petersplatz mit den denkwürdigen Worten angesprochen hat: 'Habt keine Angst! Öffnet, ja reißt die Tore weit auf für Christus!'. Was der neugewählte Papst von allen erbat, das hat er selbst als 
erster vorgelebt: Er hat die Gesellschaft, die Kultur, die Bereiche der Politik und der Wirtschaft für Christus geöffnet. Mit der Kraft eines Riesen - die er von Gott erhalten hat - hat er eine Tendenz umgedreht, die unumkehrbar erscheinen mochte. Mit seinem Zeugnis des Glaubens, der Liebe und des apostolischen Mutes, das von einer großen Menschlichkeit begleitet wurde, hat dieser beispielhafte Sohn der polnischen Nation den Christen auf der ganzen Welt geholfen, keine Angst zu haben, sich Christen zu nennen, zur Kirche zu gehören und vom Evangelium zu sprechen. Mit einem Wort, er hat uns geholfen, keine Angst vor der Wahrheit zu haben, denn die Wahrheit ist die Garantie der Freiheit. Noch einmal ganz kurz, er hat uns die Kraft wiedergegeben, an Christus zu glauben, weil Christus Redemptor hominis, der Erlöser des Menschen ist - das Thema seiner ersten Enzyklika und der Leitgedanke aller anderen"4.

${ }^{4}$ Predigt Benedikts XVI. anlässlich der Seligsprechung Johannes Pauls II., Vatikan, 1. Mai 2011, http://www.vatican.va/holy_father/benedict_xvi/homilies/2011/documents/hf_ben-xvi_ hom_20110501_beatificazione-gpii_ge.html (19.08.2011). 